\title{
Associations between TCF4 Gene Polymorphism and Cognitive Functions in Schizophrenia Patients and Healthy Controls
}

\author{
Xianghua Zhu ${ }^{1,5}$, Huang Gu ${ }^{2,5}$, Zhen Liu ${ }^{2}$, Zhansheng $\mathbf{X u}^{2}$, Xiongying Chen ${ }^{2}$, Xiaochen Sun ${ }^{2}$, Jinguo Zhai ${ }^{3}$, \\ Qiumei Zhang ${ }^{3}$, Min Chen ${ }^{3}$, Keqin Wang ${ }^{3}$, Xiaoxiang Deng ${ }^{2}$, Feng Ji ${ }^{3}$, Chuanxin Liu ${ }^{1,3}$, Jun Li*,2, Qi Dong ${ }^{2}$ and \\ Chuansheng Chen ${ }^{4}$
}

'People's Dong-fang Hospital of Xuzhou, Xuzhou, China; ²State Key Laboratory of Cognitive Neuroscience and Learning, Beijing Normal University, Beijing, China; ${ }^{3}$ School of Mental Health, Jining Medical University, Jining, China; ${ }^{4}$ Department of Psychology and Social Behavior, University of California, Irvine, CA, USA

The SNP rs2958 82 was reported to be significantly associated with schizophrenia (SCZ) in Han Chinese. This study examined this SNP's associations with cognitive functions in 580 SCZ patients and 498 controls. Cognitive functions were assessed using the Wechsler Adult Intelligence Scale-Revised (WAIS-RC), the Attention Network Task (ANT), the Stroop task, the dot pattern expectancy (DPX), task and the $\mathrm{N}$-back working memory task. Results showed significant or marginally significant interaction effects between genotype and diagnosis status on IQ $(P=0.01 \mathrm{I})$ and attention-related tasks (ie, the forward digit span of WAIS-RC, $P=0.005$; the ANT conflict effect; $P=0.020$, and its ratios over mean reaction time $(R T), P=0.036$; the Stroop conflict effect, $P=0.032$, and its ratios over mean $R T$, $P=0.062$; and the DPX task's error rate under the BX condition, $P<0.001$, and the error rate of $B X$ minus the error rate of $A Y$ $(B X-A Y), P=0.002)$. There were no such interaction effects on the measures of working memory (all $P$-values $>0.05)$. Further analysis of the significant genotype-by-diagnosis interactions showed that the risk $(T)$ allele was associated with better performance on cognitive tasks in patients but with worse performance in controls. These results seem to indicate that the association between this SNP and selected cognitive functions may be of an inverted $U$-shaped pattern. Future research is needed to replicate these results and to explore the biochemical mechanisms behind this association.

Neuropsychopharmacology (2013) 38, 683-689; doi:I0.I038/npp.2012.234; published online 19 December 2012

Keywords: schizophrenia; attention; working memory; TCF4; polymorphism

\section{INTRODUCTION}

In one of the largest genome-wide association studies (involving 2663 schizophrenia (SCZ) patients and 13498 controls) and its follow-up study (involving 4999 SCZ patients and 15555 controls), Stefansson and colleagues (Stefansson et al, 2009; Steinberg et al, 2011) identified the basic helix-loop-helix (bHLH) transcription factor (TCF4) gene as one of the most consistent and significant susceptibility genes for SCZ. A recent study in Han Chinese population using 2496 SCZ patients and 5184 healthy controls also supported that finding (Li et al, 2010). TCF4 is critical for normal brain development (Flora et al, 2007). Human haploinsufficiency of TCF4 may result in severe

*Correspondence: Dr J Li, State Key Laboratory of Cognitive Neuroscience and Learning, Beijing Normal University, 19\# Xinjiekouwai Road, Beijing 100875, China, Tel: + 8610 58801755, Fax: + 861058801755 , E-mail: lijundp@bnu.edu.cn

${ }^{5}$ These authors contributed equally to this work.

Received 25 June 20 12; revised 9 October 20 I2; accepted 31 October 2012; accepted article preview online 9 November 2012 cognitive deficiency (ie, mental retardation) in patients with Pitt-Hopkins syndrome (Pitt and Hopkins, 1978) or Angelman syndrome (Takano et al, 2010). However, the role of the TCF4 gene in cognitive deficiency in SCZ patients has seldom been studied (for exceptions, see Lennertz et al (2011); Quednow et al (2011)).

In an animal study, transgenic mice overexpressing the TCF4 gene in the brain showed reduced prepulse inhibition (PPI) (Brzozka et al, 2010), a core trait of human SCZ. A study in humans further suggested that the SCZ risk allele (C) of rs9960767 in this gene was significantly associated with reduced PPI in both healthy controls and patients with SCZ spectrum disorder (Quednow et al, 2011). Reduced PPI is an important intermediate phenotype of SCZ and is believed to be modulated by attention as assessed by the continuous performance test (CPT) (Hazlett et al, 2001, 2008), the attention network test (ANT) (Sobin et al, 2005) and the Stroop test (Molina et al, 2009). However, the role of the TCF4 gene in the etiology of attention function has not been studied.

In the Han Chinese population, rs9960767 is not polymorphic. Very close to rs9960767 $(\sim 6 \mathrm{kbp})$ and in the same intron (intron 4) is another SNP (rs2958182) that 
is in complete linkage disequilibrium with rs9960767 in the HapMap European samples. This SNP is polymorphic among Chinese and has been suggested as a risk SNP for SCZ (Li et al, 2010). However, the reported risk allele of rs2958182 was the major allele (T), not the minor allele (A) that was linked to the risk C allele of rs9960767 for Europeans. In sum, the same gene appears to be relevant for SCZ in both Europeans and Chinese, but the specific risk alleles were different. It is imperative to further explore the role of rs2958182 in China.

The current study aimed to explore the function of rs2958182 by testing its association with PPI-modulated (attention-related) cognitive functions. The attentionrelated cognitive tasks included the ANT, the Stroop test, the dot pattern expectancy task (DPX, a revised version of AX-CPT), and the Wechsler forward digit span task. Because some of these tasks rely on both working memory and attention, we included two typical working memory tasks (an N-back task and the Wechsler backward digit span task) as covariates to partial out working memory in order to focus on attention functions. We hypothesized that the risk allele (T) of rs2958182 would be associated with reduced ability of attention as reflected by a strong conflict effect of the ANT and the Stroop test, high error rates on the DPX task, and low scores on the forward digit span task.

\section{MATERIALS AND METHODS}

\section{Subjects}

The samples consisted of 580 patients with SCZ and 498 healthy controls. All subjects were Han Chinese. The patients were recruited between August 2008 and October 2011, from the inpatients of the Ankang Hospital in Shandong Province, a division of the Jining Medical College. All patients had been hospitalized for $<1$ month and fulfilled the ICD-10 criteria for SCZ based on the diagnostic consensus of two experienced psychiatrists using the Mini International Neuropsychiatric Interview. This scale has a Chinese version with high reliability and validity ( $\mathrm{Si}$ et al, 2009). Subjects were excluded if one of the psychiatrists was uncertain about a given patient's diagnosis. The general recruitment procedure was that a clinician first judged if the patient satisfied the inclusion and the exclusion criteria (see below). The clinician and the psychologist then together explained the study to the patient, including drawing of blood and cognitive tests, and answered any question the patient had. Subjects then signed the informed consent document. Afterwards, blood was drawn and the psychologist administered the cognitive tests to the patient.

The positive and negative syndrome scale was used to assess each SCZ patient's positive (SAPS) and negative (SANS) symptoms at the time of the administration of the cognitive tests. The mean score of the patients' SAPS was $18.77 \pm 6.76$ and the mean SANS score was $17.32 \pm 7.43$. The mean age of onset was $22.83 \pm 8.28$ and the mean duration of illness was $5.49 \pm 7.21$ years. All patients were undergoing monotherapy with atypical antipsychotics and had been treated for $>2$ weeks. Exclusion criteria for the patients included a history of other psychiatric disorders, a history of severe head injury (including any closed or open head injuries that may be related to current symptoms or impact cognitive functions), currently having acute psychotic episodes, current substance abuse, and failure to cooperate during the cognitive tests. Subjects were deemed by the experimenter as 'fail to cooperate' when they abruptly stopped performing tasks in the middle of the experiment, when they pressed keys only when prompted by the experimenter, and when they failed to cooperate to complete the practice trials of a test to reach an acceptable threshold of accuracy after multiple attempts.

The healthy controls were from the same geographical region as the patients and were interviewed by experienced psychiatrists to screen for any personal or family history of psychiatric disorders. Additional demographic information for both patients and the healthy controls is shown in Table 1. This study was approved by the Institutional Review Board of the Institute of Cognitive Neuroscience and Learning at Beijing Normal University, and all subjects gave written informed consent for this study.

\section{SNP Genotyping}

Genomic DNA was extracted from a $200 \mu$ EDTA venous blood sample from each subject using the QuickGeneMini80 equipment and QuickGene DNA whole blood kit S (Fujifilm, Tokyo, Japan). To genotype rs2958182, the forward primer (5'-CTACTCATGTAACTCTCCACTT- $\left.3^{\prime}\right)$ and the reverse primer $\left(5^{\prime}\right.$-CCATACTTGCTCATTG TAACTG-3 ${ }^{\prime}$ ) were designed using the software Primer 6.0. PCR was performed using 9700 thermocycler (Applied Biosystems, Foster City, CA, USA) in $20 \mu \mathrm{l}$ amplification mixture containing $0.5 \mu \mathrm{M}$ of each primer, $200 \mu \mathrm{M}$ dNTPs (Fermentas, Burlington, VT, USA), 1U Taq polymerase (Fermentas), $1 \times$ Taq buffer and 30 ng template DNA. After initial $5 \mathrm{~min}$ at $96^{\circ} \mathrm{C}$, the PCR proceeded with 36 cycles of $96{ }^{\circ} \mathrm{C}$ for $30 \mathrm{~s}, 51{ }^{\circ} \mathrm{C}$ for $30 \mathrm{~s}$, and $72{ }^{\circ} \mathrm{C}$ for $30 \mathrm{~s}$. After a final $10 \mathrm{~min}$ at $72^{\circ} \mathrm{C}$, the PCR was terminated at $4{ }^{\circ} \mathrm{C}$. Then $5 \mu \mathrm{l}$ amplified products were digested with $10 \mathrm{U}$ restriction enzyme Tsp509I (Fermentas) overnight and followed by electrophoresis in 3\% agarose gels. The AA genotype can be identified by the presence of a single band of $255 \mathrm{bp}$, and the TT genotype can be identified by two bands: 99 and $156 \mathrm{bp}$, and the AT genotype had all the above three bands: 255, 156 , and $99 \mathrm{bp}$. Each genotype was read by two individuals independently. The success rate for this SNP is above $95 \%$ and the reproducibility of the genotyping was $100 \%$ according to a duplicate analysis of $20 \%$ of the genotypes.

\section{Cognitive Tasks}

Among all the subjects with genotype information on rs2958182, 526 patients and 421 controls completed the

Table I Differences in the Demographic Data Among Patients with SCZ and Healthy Controls

\begin{tabular}{lcccc}
\hline & SCZ & Controls & F or $\chi^{2}$ & $\boldsymbol{P}$-value \\
\hline Gender (female/male) & $183 / 343$ & $161 / 260$ & 1.204 & 0.272 \\
Age (years) & $28.150 \pm 7.864$ & $22.990 \pm 7.055$ & $\mid 10.29$ & $<0.00 \mid * * *$ \\
Education (years) & $9.990 \pm 2.886$ & $10.630 \pm 2.789$ & $\mid 1.58$ & $0.00 \mid * *$ \\
\hline
\end{tabular}

** $P$-value $<0.05$. 
Wechsler Adult Intelligence Scale-Revised (WAIS-RC), including the forward and backward digit span (FDS and BDS) tasks. Almost all healthy controls finished the ANT (402), Stroop task (404), DPX task (403), and N-back task (399), but only a subsample of the patients finished the same set of tasks (383 for the ANT task, 197 for the Stroop task, 180 for the DPX task, 375 for the 1-back task, and 366 for the 2-back task) because these tasks were added later to data collection from the patients.

The DPX task was first introduced by MacDonald et al (2005) and was shown to have adequate reliability (Jones et al, 2010). The DPX task in this study was presented in 4 blocks of 40 stimulus pairs (the cues and the probes) that were presented sequentially in the center of a 14-inch screen. The stimuli were Braille font dot patterns and were presented in pairs (cue-probe). There were four conditions: AX, AY, BX, and BY, where A and B designate different cues and $\mathrm{X}$ and $\mathrm{Y}$ different probes. After $350 \mathrm{~ms}$ of a fixation cross, a cue was presented for $1000 \mathrm{~ms}$, followed by a $4000 \mathrm{~ms}$ delay, and then the probe was presented for $500 \mathrm{~ms}$. Between trials was a $650 \mathrm{~ms}$ white intermission screen. Subjects were instructed to press a target key in the AX condition but to press the non-target key in all other conditions (see MacDonald et al, 2005 for details). As in MacDonald et al (2005) study, the 40 trials of each block consisted of $28(70 \%)$ AX trials, $5(12.5 \%)$ AY trials, 5 (12.5\%) BX trials, and 2 (5\%) BY trials. The error rate of the $\mathrm{BX}$ and $\mathrm{BX}-\mathrm{AY}$ (the error rate of $\mathrm{BX}$ minus the error rate of AY) was used as the main measurements because previous studies showed that, compared with healthy controls, both SCZ patients and their healthy relatives showed significantly higher error rates in $\mathrm{BX}$ condition and a tendency of making less $\mathrm{AY}$ than $\mathrm{BX}$ errors thus higher value of $\mathrm{BX}-\mathrm{AY}$ (MacDonald et al, 2003, 2005).

The ANT uses a central arrow and four flanker arrows as stimuli. Subjects were required to respond to the direction of the central arrow while ignoring all flanker arrows. More detailed descriptions of the design of this task can be found elsewhere (Fan et al, 2002). The current study used the short version of this test, which contained 144 trials and can be freely downloaded from Fan's webpage (http://www.sacklerinstitute.org/users/jin.fan/). This version omitted the double cue conditions and the neutral target conditions, which were irrelevant to the calculation of the conflict effect. The conflict effect was calculated by subtracting the mean RT of all correct trials for the congruent condition (target and flanker arrows pointing the same direction) from the mean RT of correct trials for the incongruent condition (target and flanker arrows pointing different directions). Following the suggestion of Fan et al (2001), ratios of the conflict effect were also calculated (conflict effect/mean RT) to take into account the direct or indirect effects of overall mean RT. The conflict effect was reported to be reliable with test-retest reliability of 0.77 (Fan et al, 2001).

We also used a computerized classic Stroop task, which had been shown to have similar reliability as the card version (Siegrist, 1995). Color words were presented in three different ink colors: red, green, and blue. The subjects were asked to press one of the three keys to indicate the color of ink while ignoring the meaning of the color word. The ink color and the word's meaning may be congruent or incongruent. The conflict effect was measured, similar to the ANT, by subtracting the mean RT of all correct trials for the congruent target condition from the mean RT of correct trials for the incongruent target condition. The ratios of this conflict effect over mean RT were also calculated.

The N-back tasks have been widely used to test working memory. Previous research has shown that these tasks have high test-retest reliability at about 0.7 (Studer-Luethi et al, 2012). The N-back task of this study was similar to the version introduced by Callicott et al (1998). In this task, a white circle was presented randomly at one of the four corners of a gray diamond-shaped square in white background on an IBM 14-inch screen notebook. The four response buttons were arranged also in a diamond shape similar to the configuration of the white circles presented on the screen. Subjects used their right index or middle finger to press one of the four buttons to match the target stimulus. There were three task conditions: $0-, 1-$, and 2-back. In the 0-back task, the subjects were instructed to press the button whose position was the same as the white circle on the screen for the current trial. In the 1-back task, the subjects pressed the button corresponding to the position of the white circle presented 1 trial before the current one. In the 2-back task, the subjects pressed the button corresponding to the position of the white circle presented 2 trials before the current one. Each condition (performed in one block) included 48 trials. All subjects followed the order of 0-, 1-, and 2-back conditions. The stimulus presentation time was $200 \mathrm{~ms}$ and the inter-stimulus interval was $800 \mathrm{~ms}$. Following Blokland et al's twin study (Blokland et al, 2008), the current study used error rate (percentage of wrong responses) of both 1-back and 2-back trials to index the performance.

\section{Analysis}

The Hardy-Weinberg test of the SNP was done by using the PLINK program (Purcell et al, 2007). All other analyses were done by using SPSS version 13.0 (SPSS, Chicago, IL, USA). Non-genetic factors, including age, gender, and years of education among genotypic groups were compared using either one-way ANOVA or $\chi^{2}$-test. Owing to the small number of the AA genotype (11 in the control group and 8 in the SCZ group in the total sample), we combined the AA genotype with the AG genotype. Preliminary analysis showed that all cognitive performances were normally or almost normally distributed (kurtosis and skewness for all cognitive measures could be found in Supplementary Table S1) with constant variances (Levene test across groups (genotype, diagnosis) showed that all $P$-value $>0.05$ ). The associations between cognitive function and the SNP were tested by two-way ANCOVA. The two fixed factors were genotype (minor allele [A] carriers vs major allele [T] homozygotes) and diagnosis (SCZ vs controls). Demographic factors including age, gender, and years of education were entered as covariates. Significant interaction effects between genotype and diagnosis or significant main effects of genotype were followed up with the simple effects analysis of genotype in patients and controls separately. In the simple effects analysis, age, gender, and education years were also used as covariates. 


\section{RESULTS}

The allele frequency of this SNP (with the A allele frequency was 0.112) was consistent with Li et al's study (0.103) (Li et al, 2010). No deviation from Hardy-Weinberg equilibrium was found in the total sample ( $P$-values $>0.05)$. For demographic factors, patients and controls were comparable in terms of their gender distribution, but they differed significantly in mean age and education level (both $P$-values $<0.05$, see Table 1 ). Genotype was not associated with any demographic factors or clinical characteristics either for patients or controls (all $P$-values $>0.05$, see Table 2 ).

After controlling for age, gender, and education level, the interactions between genotype and diagnosis were significant for total IQ $(\mathrm{F}=6.56, P=0.011)$, the FDS score $(\mathrm{F}=7.76, P=0.005)$, both $\mathrm{BX}(\mathrm{F}=20.64, P<0.001)$ and BX-AY $(\mathrm{F}=9.71, P=0.002)$ scores of the DPX task, both the conflict effect $(\mathrm{F}=5.45, P=0.020)$ and its ratios $(\mathrm{F}=4.41$, $P=0.036)$ of the ANT, and the Stroop conflict effect $(\mathrm{F}=4.63, P=0.032)$. The ratios of the Stroop conflict effect were marginally significant $(\mathrm{F}=3.50, P=0.062)$. The main effect of genotype was only significant for both indices from the DPX task (BX, F=11.90, $P=0.001 ; \mathrm{BX}-\mathrm{AY}, \mathrm{F}=2.75$, $P=0.098)$. No significant main effects of genotype or interactions were found for the three measures of working memory (all $P$-values $>0.05$ ) (see Table 3 ).

To further explore the significant interactions reported above, we analyzed the data separately for patients and controls. We found opposite directions of associations between genotype and cognitive functions for the two groups of subjects. In patients, four significant and one marginally significant associations were found with the $\mathrm{T}$ allele carriers showing better performance: IQ $(\mathrm{F}=6.28$, $P=0.013$ ), the DPX task (BX, $\mathrm{F}=7.44, P=0.007$; BX-AY, $\mathrm{F}=13.18, P<0.001$ ), and the Stroop task (the conflict effect,
$\mathrm{F}=3.05, \quad P=0.082$; the conflict effect ratios, $\mathrm{F}=3.93$, $P=0.049$ ). This analysis was re-run using the duration of the illness, age of onset, medication dose, and both positive and negative symptom scores as additional covariates, and the results did not change (see Supplementary Table S2). In contrast, among the controls the $\mathrm{T}$ allele carriers showed significantly worse performance on the FDS task $(\mathrm{F}=6.37$, $P=0.012)$ and the conflict effect of the ANT task $(\mathrm{F}=4.11$, $P=0.043)$.

Finally, the above analyses were re-run by including the three working memory measures as additional covariates. The significant results remained the same as before (see Supplementary Table S3), indicating that the significant associations reported above were not attributable to differences in working memory.

\section{DISCUSSION}

This study is the first to report an association between TCF4 gene polymorphism and intelligence and attention process. We found significant interaction effects between genotype and diagnosis in all four measures of attention even after controlling for working memory. It appeared that the role of TCF4 is specific to attentional processes. Further analysis showed opposite directions of associations in patients and controls. The risk $\mathrm{T}$ allele of rs 2958182 was associated with worse performance in controls, but with better performance in patients.

As mentioned in the introduction, the SCZ risk allele of rs2958182 in Han Chinese was linked to the protective allele of rs9960767 in Europe samples, which makes it difficult to relate our results to the previous studies on European samples directly. One approach is to discuss the results from the perspective that population-specific risk allele

Table 2 Differences in the Demographic Data Across TCF4 rs2958I 82 Genotype for Patients and Healthy Controls

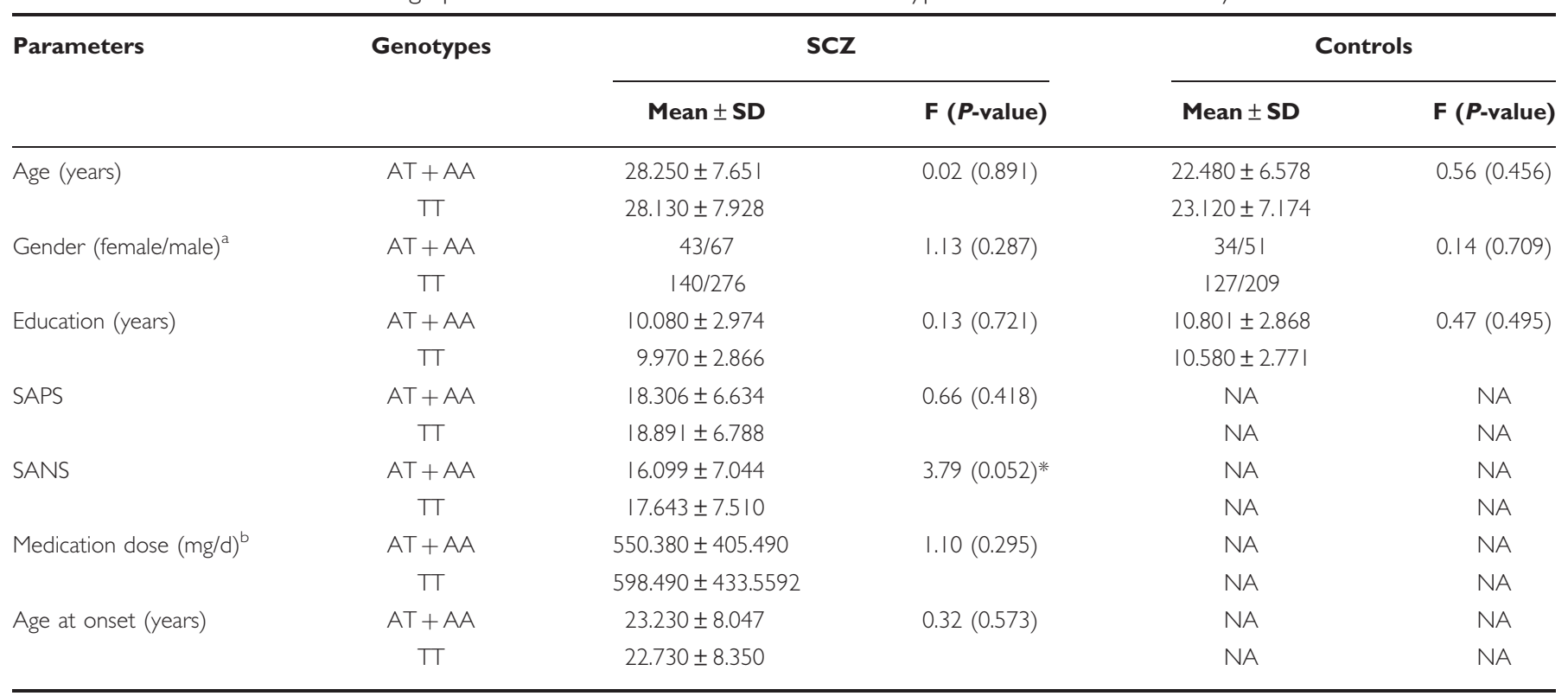

Abbreviation: NA, not applicable.

$*$ P-value $<0.1$.

a $\chi^{2}$-test.

${ }^{\mathrm{b}}$ Chlorpromazine equivalents. 
Table 3 Cognitive Functions According to TCF4 rs2958I 82

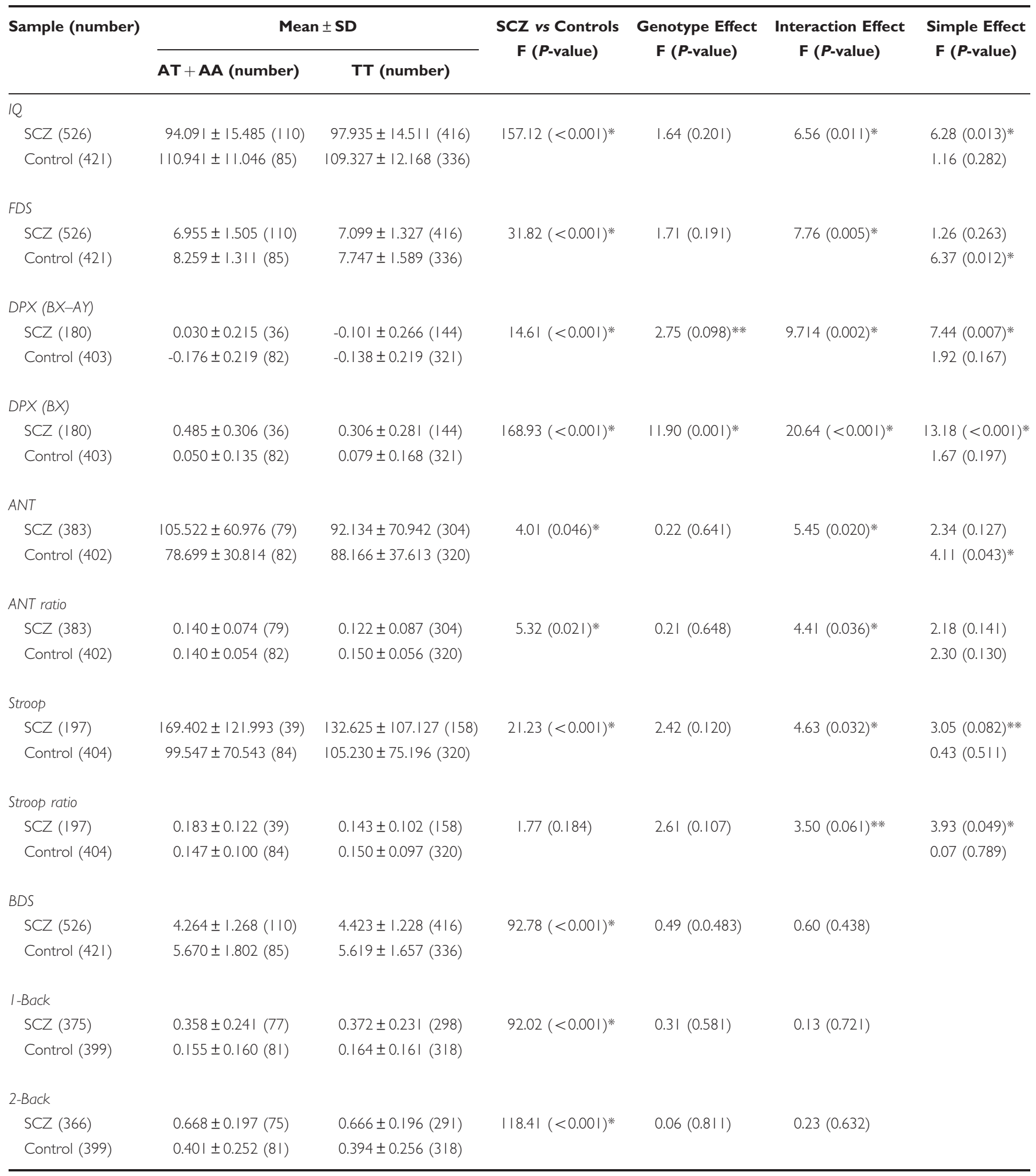

** $P$-value $<0.1$.

*P-value $<0.05$.

should be associated with deficient cognitive functions. In that sense, our results in controls were consistent with those of the only other study on rs9960767 and cognitive functions in healthy controls (Quednow et al, 2011). Both studies showed that the risk allele was associated with worse cognitive functions. However, our results in patients were 
inconsistent with those from another SCZ sample of the same study (Quednow et al, 2011). Instead, they were consistent with the results reported in another study by the same group of researchers (Lennertz et al, 2011). The latter included only SCZ patients and found that the risk allele was associated with better memory.

One interpretation of the opposite effects of TCF4 gene polymorphism on cognitive functions in patients and controls is that TCF4 may have an inverted U-shaped relation with cognitive functions. Indeed, previous studies have shown that cognitive functions are impaired when the level of TCF4 is either below or above an optimal range. For example, heterozygous deletions of the TCF4 gene in human were found to result in mental retardation (Flora et al, 2007) and overexpression of the TCF4 gene in mice resulted in reduced PPI and memory (Brzozka et al, 2010). It is possible that the risk allele of rs2958182 boosts (or normalizes) the performance in patients (at the ascending arm of the inverted-U) but worsens the performance in controls (at the descending arm of the inverted-U). Of course, this interpretation needs to be substantiated with biochemical evidence of hyper- or hypoexpression of the TCF4 gene in SCZ patients. Nevertheless, the inverted-U pattern of results has been reported for other genes such as the val/met polymorphism of the COMT gene (Barnett et al, 2008; Prata et al, 2009). Within research on SCZ patients and their controls, a SNP in the Neuregulin 3 gene also showed the same pattern of results, with the risk allele linked to better performance on a CPT task in SCZ patients but worse performance in healthy controls (Morar et al, 2011).

Another explanation for the current results is a possible interaction between TCF4 and another gene. TCF4 belongs to the bHLH transcription factor family. It can activate or repress multiple genes' expression by forming heterodimers with other bHLH transcription factors (Flora et al, 2007). Potential interactions among these genes need to be examined in future research.

Taken together, the results of existing studies suggest that the role of the TCF4 gene is complex. To help appreciate the complexity, especially the inconsistent results in patients of Quednow et al's study on PPI and ours on attention process, it is worth mentioning that a recent study in mice found that PPI and attention process as measured by five-choice serial RT task (5-CSRTT, a task similar to the human CPT task) did not share genetic factors (Loos et al, 2012). Loos et al's study further found that 5-CSRTT mapped to one locus as previously reported by Hitzemann et al (2008), but the direction of association was opposite: The allele associated with reduced PPI in Hitzemann et al (2008) was associated with better performance in 5-CSRTT task in Loos et al (2012).

Another important finding of the current study was the significant association between rs 2958182 of TCF4 and IQ in patients. Previous studies have provided clues that TCF4 may be a candidate gene for intelligence. For example, it was found that haploinsufficiency of TCF4 may induce PittHopkins syndrome that is characterized by severe mental retardation (Flora et al, 2007), and that mutations in the TCF4 gene are linked to other types of mental retardation, such as Pitt-Hopkins and Angelman syndromes (Kalscheuer et al, 2008; Takano et al, 2010). Quednow et al (2011) also found a moderate effect of rs9960767 on years of education in patients (although not in controls). The authors believed that this association reflected a possible effect of TCF4 on intellectual function (not measured in that study) because of the high correlation between education level and intelligence. However, another study of the same group (Lennertz et al, 2011) assessed patients' IQ but failed to find a significant association between rs9960767 and IQ. It is worth noting that subjects did not take the same IQ test (198 patients took a German vocabulary test for verbal intelligence, the MehrfachwahlWortschatz-Test, but the remaining 203 patients took WAIS-R), which may have confounded the results. To our knowledge, the current study is the first to report a significant association between TCF4 gene polymorphism and measured IQ. However, similar to Quednow et al (2011) results based on inferred intellectual function from years of education, our results were significant only for SCZ patients.

Some limitations of this study should be addressed. First, most tasks in this study were computerized and were somewhat difficult for some patients, especially those who were seriously impaired. Because these patients failed to complete one or more of the tasks, they had to be excluded from our analysis. Therefore, it should be cautioned that the results of this study may not be generalized to such patients. Second, some clinical and demographic factors may confound the current results. For example, patients' symptoms and current high-dose antipsychotic medication might have biased patients' performance and hence influenced the current results. Other confounding factors might include some demographic factors such as age and education levels that were not well matched between patients and controls, although they were included as covariates in our analysis. Future studies should attempt to obtain better matched samples and possibly multiple patient samples with varying dosage of medications. Finally, it is worth mentioning that the current study did not correct for multiple comparisons involving the cognitive tests. We aimed to measure the same construct with multiple cognitive measures, which were necessarily intercorrelated with one another. Using the conventional Bonferroni correction would result in overcorrection because it does not consider the interrelatedness among variables. Given a lack of current consensus about appropriate correction and for the sake of comparison across studies, we showed the results uncorrected.

In summary, the current study found a possible effect of the TCF4 gene (SNP rs2958182) on intelligence and attention function, but it was in opposite directions in patients and controls, which suggest a possible inverted U-shaped function of this gene polymorphism in Han Chinese population. Future research needs to replicate these results and investigate the biochemical mechanisms involved in the differential associations between a SNP and behavior across different groups of subjects.

\section{ACKNOWLEDGEMENTS}

This work was supported by grants from the Natural Science Foundation of China. (30800294), the Medical Science and Technology Plan of Shandong Province, China 
(2007HW037) and the Fundamental Research Funds for the Central Universities.

\section{DISCLOSURE}

The authors declare no conflict of interest.

\section{REFERENCES}

Barnett JH, Scoriels L, Munafo MR (2008). Meta-analysis of the cognitive effects of the catechol-O-methyltransferase gene Val158/108Met polymorphism. Biol Psychiatry 64: 137-144.

Blokland GA, McMahon KL, Hoffman J, Zhu G, Meredith M, Martin NG et al (2008). Quantifying the heritability of taskrelated brain activation and performance during the N-back working memory task: a twin fMRI study. Biol Psychol 79: 70-79.

Brzozka MM, Radyushkin K, Wichert SP, Ehrenreich H, Rossner MJ (2010). Cognitive and sensorimotor gating impairments in transgenic mice overexpressing the schizophrenia susceptibility gene Tcf4 in the brain. Biol Psychiatry 68: 33-40.

Callicott JH, Ramsey NF, Tallent K, Bertolino A, Knable MB, Coppola $\mathrm{R}$ et al (1998). Functional magnetic resonance imaging brain mapping in psychiatry: methodological issues illustrated in a study of working memory in schizophrenia. Neuropsychopharmacology 18: 186-196.

Fan J, McCandliss BD, Sommer T, Raz A, Posner MI (2002). Testing the efficiency and independence of attentional networks. J Cogn Neurosci 14: 340-347.

Fan J, Wu Y, Fossella JA, Posner MI (2001). Assessing the heritability of attentional networks. BMC Neurosci 2: 14 .

Flora A, Garcia JJ, Thaller C, Zoghbi HY (2007). The E-protein Tcf4 interacts with Math1 to regulate differentiation of a specific subset of neuronal progenitors. Proc Natl Acad Sci USA 104: 15382-15387.

Hazlett EA, Dawson ME, Schell AM, Nuechterlein KH (2001). Attentional stages of information processing during a continuous performance test: a startle modification analysis. Psychophysiology 38: 669-677.

Hazlett EA, Dawson ME, Schell AM, Nuechterlein KH (2008). Probing attentional dysfunctions in schizophrenia: Startle modification during a continuous performance test. Psychophysiology 45: 632-642.

Hitzemann R, Malmanger B, Belknap J, Darakjian P, McWeeney S (2008). Short-term selective breeding for high and low prepulse inhibition of the acoustic startle response; pharmacological characterization and QTL mapping in the selected lines. Pharmacol Biochem Behav 90: 525-533.

Jones JA, Sponheim SR, MacDonald AW III (2010). The dot pattern expectancy task: reliability and replication of deficits in schizophrenia. Psychol Assess 22: 131-141.

Kalscheuer VM, Feenstra I, Van Ravenswaaij-Arts CM, Smeets DF, Menzel C, Ullmann R et al (2008). Disruption of the TCF4 gene in a girl with mental retardation but without the classical PittHopkins syndrome. Am J Med Genet A 146A: 2053-2059.

Lennertz L, Rujescu D, Wagner M, Frommann I, SchulzeRauschenbach S, Schuhmacher A et al (2011). Novel schizophrenia risk gene TCF4 influences verbal learning and memory functioning in schizophrenia patients. Neuropsychobiology 63: 131-136.

Li T, Li Z, Chen P, Zhao Q, Wang T, Huang K et al (2010). Common variants in major histocompatibility complex region and TCF4 gene are significantly associated with schizophrenia in Han Chinese. Biol Psychiatry 68: 671-673.

Loos M, Staal J, Pattij T, Smit AB, Spijker S (2012). Independent genetic loci for sensorimotor gating and attentional performance in BXD recombinant inbred strains. Genes Brain Behav 11: $147-156$.

MacDonald AW III, Goghari VM, Hicks BM, Flory JD, Carter CS, Manuck SB (2005). A convergent-divergent approach to context processing, general intellectual functioning, and the genetic liability to schizophrenia. Neuropsychology 19: 814-821.

MacDonald AW 3rd, Pogue-Geile MF, Johnson MK, Carter CS (2003). A specific deficit in context processing in the unaffected siblings of patients with schizophrenia. Arch Gen Psychiatry 60: 57-65.

Molina V, Montes C, Tamayo P, Villa R, Osuna MI, Perez J et al (2009). Correlation between prepulse inhibition and cortical perfusion during an attentional test in schizophrenia. A pilot study. Prog Neuropsychopharmacol Biol Psychiatry 33: 53-61.

Morar B, Dragovic M, Waters FA, Chandler D, Kalaydjieva L, Jablensky A (2011). Neuregulin 3 (NRG3) as a susceptibility gene in a schizophrenia subtype with florid delusions and relatively spared cognition. Mol Psychiatry 16: 860-866.

Pitt D, Hopkins I (1978). A syndrome of mental retardation, wide mouth and intermittent overbreathing. Aust Paediatr $J$ 14: 182-184.

Prata DP, Mechelli A, Fu CH, Picchioni M, Toulopoulou T, Bramon E et al (2009). Epistasis between the DAT 3' UTR VNTR and the COMT Val158Met SNP on cortical function in healthy subjects and patients with schizophrenia. Proc Natl Acad Sci USA 106: 13600-13605.

Purcell S, Neale B, Todd-Brown K, Thomas L, Ferreira MA, Bender $\mathrm{D}$ et al (2007). PLINK: a tool set for whole-genome association and population-based linkage analyses. Am J Hum Genet 81: 559-575.

Quednow BB, Ettinger U, Mossner R, Rujescu D, Giegling I, Collier DA et al (2011). The schizophrenia risk allele C of the TCF4 rs9960767 polymorphism disrupts sensorimotor gating in schizophrenia spectrum and healthy volunteers. J Neurosci 31: 6684-6691.

Si TM, Shu L, Dang WM, Su YA, Chen JX, Dong WT et al (2009). Evaluation of the reliability and validity of Chinese version of the MINI. International neuropschiatric interview in patients with mental disorders. Chin Mental Health J 23: 493-497 (in Chinese).

Siegrist M (1995). Reliability of the stroop test with single-stimulus presentation. Percept Mot Skills 81: 1295-1298.

Sobin C, Kiley-Brabeck K, Karayiorgou M (2005). Associations between prepulse inhibition and executive visual attention in children with the 22q11 deletion syndrome. Mol Psychiatry 10: 553-562.

Stefansson H, Ophoff RA, Steinberg S, Andreassen OA, Cichon S, Rujescu D et al (2009). Common variants conferring risk of schizophrenia. Nature 460: 744-747.

Steinberg S, de Jong S, Andreassen OA, Werge T, Borglum AD, Mors $\mathrm{O}$ et al (2011). Common variants at VRK2 and TCF4 conferring risk of schizophrenia. Hum Mol Genet 20: 4076-4081.

Studer-Luethi B, Jaeggi SM, Buschkuehl M, Perrig W (2012). Influence of neuroticism and conscientiousness on working memory training outcome. Pers Indiv Differ 53: 44-49.

Takano K, Lyons M, Moyes C, Jones J, Schwartz CE (2010). Two percent of patients suspected of having Angelman syndrome have TCF4 mutations. Clin Genet 78: 282-288. 